\title{
Key effects on the confinement improvement of the AUG hybrid scenario
}

Xiang Jian ${ }^{1,2}$, Vincent S Chan ${ }^{3,4}$, Jiale Chen ${ }^{* 5}$, Alexander Bock ${ }^{6}$, Hartmut Zohm ${ }^{6}$, Emiliano Fable ${ }^{6}$, Maximilian Reisner ${ }^{6}$, Wenfeng Guo ${ }^{5}$, Ge Zhuang ${ }^{* 3}$

1 State Key Laboratory of Advanced Electromagnetic Engineering and Technology, School of Electrical and Electronic Engineering, Huazhong University of Science and Technology, Wuhan 430074, People's Republic of China

2 Center for Energy Research, University of California, San Diego, La Jolla, California 92093-0417, USA

3 School of Physical Sciences, University of Science and Technology of China, Hefei 230026, China

4 General Atomics, PO Box 85608, San Diego, CA 92186-5608, USA

5 Institute of Plasma Physics, Chinese Academy of Sciences, Hefei 230031, China

6 Max-Planck-Institut für Plasmaphysik, Boltzmannstr. 2, 85748 Garching, Germany

Corresponding Author *, chen@ipp.ac.cn; and gezhuang@ustc.edu.cn;

Abstract. Transport analysis of a fully non-inductive high performance ASDEX Upgrade (AUG) hybrid mode discharge (A. Bock, Nuclear Fusion, 2017) is performed using a gyro-fluid transport code and a gyro-kinetic stability code. It is shown that the confluence of several mechanisms is essential for the improvement in core confinement. In addition to rotation shear, low magnetic shear, which is a common characteristic near the magnetic axis of a hybrid scenario, is found to be favorable for both electromagnetic and $\alpha$ stabilization. Together they facilitate the conditions for fast ions to establish a strong local ITB by providing additional dilution effect and enhancement of $\alpha$ stabilization. This study provides a complete picture and demonstrates that a quasilinear turbulent model is capable of quantitatively matching the experimental temperature profiles across the entire core of a hybrid mode discharge.

\section{Introduction}


High-performance H-mode is favorable for future fusion steady-state tokamak operation as it can increase the bootstrap current fraction $f_{b s}$, which leads to a reduction in the auxiliary power required for driving current (CD). It increases the recirculating gain $\left(\equiv P_{f u s} / P_{C D}\right)$, which is crucially important for the economic operation of a fusion reactor. The hybrid and reversed shear scenarios are shown to be promising candidates for the desired high performance operation[1]. The reversed shear scenario normally employs high $q$ (safety factor) operation, which is favorable for the increase of $f_{b s}\left(f_{b s} \sim q \beta_{N}\right.$ where $\beta_{N}$ is the normalized $\left.\beta\right)$ and thus makes it a preferred option for fully non-inductive operation[2]. The hybrid scenario is initially proposed to maximize the neutron fluence for the purpose of testing the design of reactor components $[1,3]$ due to its characteristics of stationary current density profile and long duration. However, recent encouraging results from DIII-D [4] and AUG [5] have shown that fully non-inductive state can also be obtained using the hybrid scenario with the current roughly equally coming from external current drive and self-driven bootstrap current. For many current drive schemes, central deposition, which is employed in hybrid scenario, is found to be most efficient since trapped particle effect is minimized and the temperature profile is more peaked than the density profile[4]. Due to the higher current drive efficiency, extrapolation of the DIII-D fully non-inductive hybrid scenario to ITER can similarly obtain fusion gain $Q$ $\sim 5[4]$. More recently, such a scenario is also proven to be compatible with ELM suppression which makes it more reactor relevant [6].

In practice, robustness of entry and easy sustainment [1] are two attractive features for the hybrid scenario. The hybrid scenario is characterized by a flat current density profile and thus a broad region of weak magnetic shear in the deep core region. However, the experimental core current profile does not always agree well with the modelled one. Specifically, the modelled current is likely to be more peaked and $q_{\min }$ $<1$ is expected, while $q_{\min }>1$ is kept in experiments. Anomalous current broadening, also referred to as 'flux pumping' $[7,8]$, has been proposed to explain such a discrepancy. It's a robust self-organizing behavior which can 'anomalously' flatten the 
current density profile in the deep core region and keeps $q_{\min }>1$, thus preventing the deleterious sawteeth from seeding and destabilizing the disruptive neoclassical tearing mode (NTM). Tokamak operation can benefit from such a mechanism since the current profile, including the ohmic and bootstrap current as well as the current driven by external systems, do not have to be carefully aligned to sustain the desired $q$ profile. Many experiments have demonstrated that the entire current profile can be kept stationary and $q_{\min }>1$ is robustly maintained over a wide range of both deposition location and external current drive power due to the existence of 'flux pumping' [4].

Good core confinement, i.e. a steep gradient in the ion temperature channel $\left(T_{i}\right)$, is frequently observed in the hybrid scenario [9]. Since the value of $a / L_{T i}\left(=a \nabla T_{i} / T_{i}\right)$ exceeds by more than $50 \%$ that of a standard $\mathrm{H}$-mode profile, we call this region an internal transport barrier (ITB) in the following discussion. Such a $T_{i}$ ITB is expected to beneficially enhance fusion power in future burning plasma device. However, the transport mechanism behind the ITB is still not well understood, which creates large uncertainties when extrapolating the hybrid scenario to ITER and DEMO reactors. Various mechanisms responsible for the ITB creation have been proposed, e.g. low magnetic shear and strong rotation have been identified as necessary conditions in experiments[10]; and nonlinear turbulence stabilization by fast ions leading to improved confinement has been found by nonlinear gyrokinetic simulation $[11,12]$. Although these findings are suggestive of the mechanisms behind the hybrid mode, they do not in isolation describe a complete picture of how different physical mechanisms work in concert to create the improved confinement in a hybrid mode discharge. In our work, we will show by quantitative analysis (i) the dominant factors in confinement enhancement in different radial regions, and (ii) how they work integrally to form the distinctive transport profiles observed in experiments. Our analysis across the core plasma is critical in quantitatively reproducing the measured temperature profiles.

This paper will be organized as follows. The main characteristics of the AUG hybrid scenario that this paper focuses on is described in section 2. Simulation setup 
is introduced in section 3 and section 4 will present the simulation results and detailed analysis. The paper is summarized in section 5 .

\section{AUG hybrid scenario}

The hybrid scenario has been extensively studied on AUG[13], which lays a firm foundation for its extrapolation to $\operatorname{ITER}[1,14]$. The elaborated transport analysis reported here is based on a recent representative high performance AUG hybrid shot (\#32305) [5] from the 2016 campaign, which is also fully non-inductive. AUG has been converted into a fully-tungsten machine, thus making the hybrid scenario in AUG more reactor-relevant. Unlike some hybrid shots that need early heating in the plasma ramp-up phase, this shot does not strongly rely on the precise time-scheme control of early heating, which eases the access of hybrid scenario in practice. For this shot, both the electron cyclotron (EC) wave and neutral beam injection (NBI) are applied only after the plasma current $I_{p}$ has achieved flat top $(t \geqslant 1 \mathrm{~s}$, fig 1 (a)). Both current driven by EC wave (ECCD) and NB (NBCD) are used to tailor the current profile, while additional NB power is used to improve the $\beta$ value. The target $\beta_{N}$ $(\sim 2.7)$ is achieved at $t \sim 3.5 \mathrm{~s}$ by gradually increasing the NB power and is maintained via feedback control of the NBI power from then on till $t \sim 5 \mathrm{~s}$, at which time the plasma starts to ramp down. During flat-top $(t \sim[3.5 \mathrm{~s}, 5 \mathrm{~s}])$, the surface loop voltage $V_{\text {loop }}$ approaches 0 (fig 1(c)), which can be seen in fig 1(e) showing the external driven current and bootstrap current components work in concert to eliminate the ohmic current to achieve a fully non-inductive state. We also call attention that the central ion temperature $\left(T_{i}\right)$ deviates strongly from the electron temperature $\left(T_{e}\right)$ (fig 1(b)). 


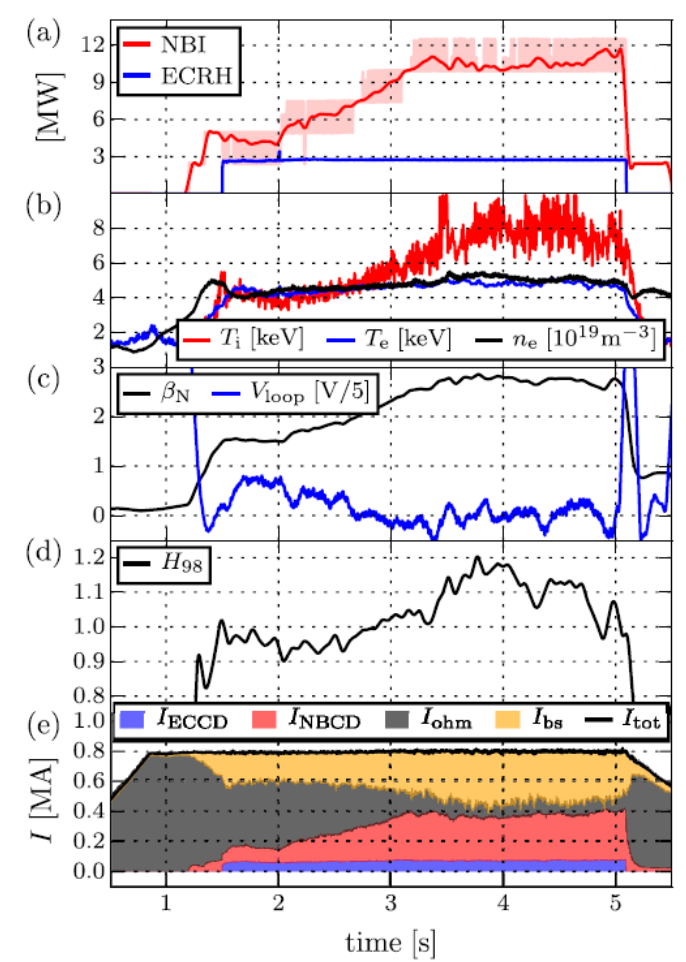

Figure 1. (a) Heating trajectories; (b) Evolution of central temperature and line-averaged electron density; (c) Evolution of $\beta_{N}$ (black) and plasma surface voltage $V_{\text {loop }}$ (blue); (d) Evolution of $H_{98}$; (e) Composition of toroidal plasma current; total current $I_{p}$, bootstrap current $I_{b s}$ as calculated by IDE, $I_{E C C D}$ from TORBEAM, $I_{N B C D}$ from TRANSP/NUBEAM. Reprinted from figure 5 of Ref[5].

In the flat-top regime of plasma performance (e.g., $t \sim 4.0 \mathrm{~s})$, the plasma current $I_{p}$ $=800 \mathrm{kA}$ and $B_{t}=2.5 \mathrm{~T}$ so that $q_{95}=5.3$ and poloidal $\beta\left(\beta_{p}\right)$ reaches 2.0 . Both the $q_{95}$ and $\beta_{p}$ values are highly relevant for high performance, steady-state operation. The line averaged density $\left\langle n_{e}\right\rangle \sim 6 \cdot 10^{19} m^{-3}\left(\sim 0.5 n_{G W}\right)$, which is low compared to the typical hybrid scenario[1] $\left(\sim 0.85 n_{G W}\right)$. The relatively low density is favorable for the increase of both the bootstrap current fraction due to the lower collisionality and high current drive efficiency in order to achieve the fully non-inductive state. EC waves launched from low field side deposit their powers near axis to efficiently drive the current there, while both on-axis and off-axis NB are used. The corresponding current profiles are shown in figure 2(a), with the ohmic current approaching 0 as a whole, indicating a nearly fully non-inductive state, which is consistent with fig 1(c) and (e). 
The resultant $q$ profile for $\rho<0.4$ is quite flat with $q_{\min } \sim 1.2$ (fig 2(b)), where $\rho$ is the normalized toroidal flux. The total pressure and fast ion pressure are over-plotted in fig 2(b). It is noted that significant fast ion pressure induced by strong neutral beam heating exists over a broad core region. The only impurity ion is tungsten (W) with its concentration $n_{w} / n_{e} \sim 10^{-5}$.

We refer those readers who are interested in more details about this shot to $\operatorname{Ref}[5]$. We further note that there could be some inconsequential differences of profile details between $\operatorname{Ref}[5]$ and this paper since we reconstructed the equilibrium using the AUG experimental data with $\operatorname{EFIT}[15,16]$ while the equilibrium information in $\operatorname{Ref}[5]$ is given by IDE[17]. In addition, based on the EFIT equilibrium and AUG auxiliary heating setup information, the power deposition and current drive is also calculated independently using the ray tracing code TORAY [18] and the Monte-Carlo code NUBEAM [19] for ECH and NBH, respectively (ECCD and NBCD profiles are shown in fig 2(a)). By using different tools by different groups to reproduce the equilibrium, current drive and power deposition shown in this paper, we have checked that satisfactory consistency has been met.
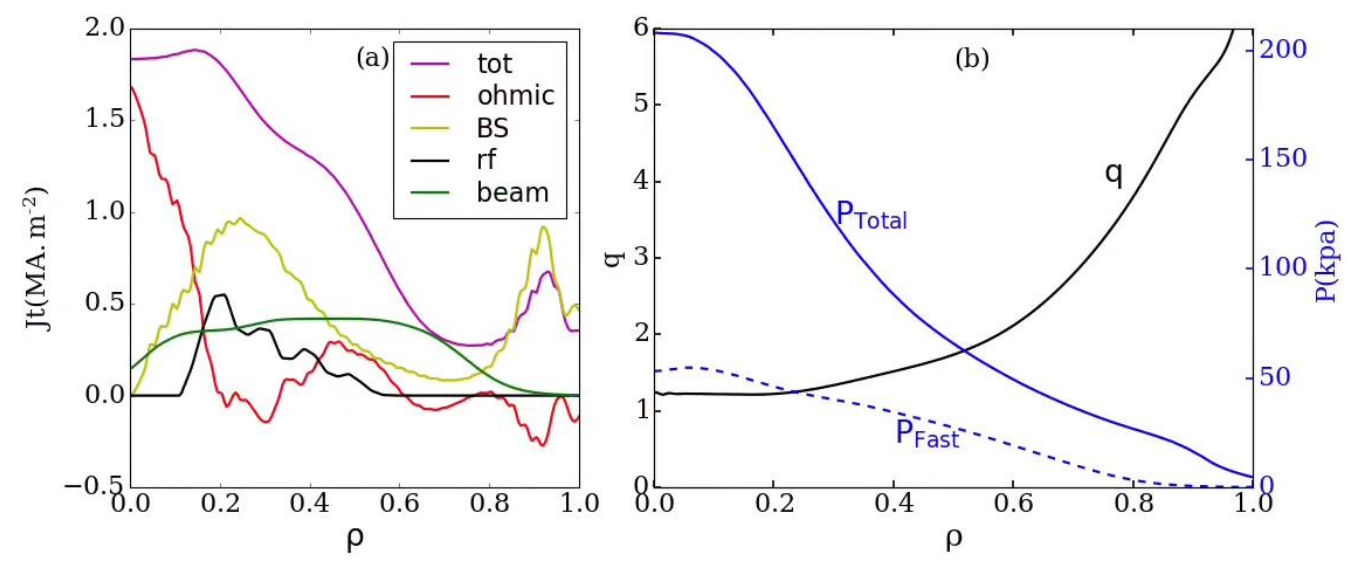

FIG. 2. (a) Profiles of difference current component,'tot','ohmic','BS','rf'and 'beam' denotes the total, ohmic, bootstrap, ECCD and NBCD, respectively; (b) Experimental $q$ profile, total pressure and fast ion pressure profile are represented by black, blue and blue dashed line, respectively

\section{Simulation Setup}


In order to understand the transport mechanism for such a hybrid scenario, especially for the formation of $T_{i} \mathrm{ITB}$, which is frequently observed in the core region, the TGYRO[18] code is employed for the transport simulation of core temperature profiles. It calls the theory-based quasi-linear transport model TGLF-SAT0 [19] and first-principle drift kinetic code $\mathrm{NEO}[20]$ for turbulent and neoclassical transport flux calculations, respectively. NEO is a first-principles code that directly solves the drift-kinetic equation to ensure a high accuracy in neoclassical transport. It has been extensively benchmarked with analytical theories and codes like NCLASS[21]. TGLF is a widely used gyro Landau fluid (GLF) quasi-linear transport model with improved comprehensive physics compared to its predecessor, GLF23 [22] and has been systematically verified against the GKS[23] gyrokinetic code in linear calculation. The turbulence saturation intensity is fitted to a database of nonlinear $\operatorname{GYRO}[24,25]$ gyrokinetic simulations with Miller geometry[26], which is a local equilibrium model characterized by nine parameters, including magnetic shear, local pressure gradient etc, and is used throughout this paper. The local equilibrium is always self-consistently satisfied when one or more of the nine parameters is changed. TGLF has been validated against a database of over 100 discharges [19].

TGYRO iteratively finds a set of kinetic gradients that drive the transport flux that can match the target flux, so that transport timescale steady state profile, which should always be satisfied in a stationary discharge state, can be obtained. The target flux is determined by the volume integral of the sum of external heating and radiation power. The simulated profiles can therefore be obtained by integrating the kinetic gradients over radius. We fix the boundary at $\rho_{b d r y}=0.7$, outside which location the temperatures are fixed to experiment and not evolved, i.e. no attempt is made to model the edge pedestal. The bremsstrahlung, cyclotron, and line radiation calculations are based on models in $\operatorname{Ref}[27]$.

Our approach is to systematically introduce relevant physics in the theoretical models to produce a temperature profile that best matches the measurement inside the 
boundary. From the results we ascertain the dominant mechanisms.

\section{Simulation results and analysis}

\subsection{Simulation results}

Simulations of $T_{i}$ and $T_{e}$ profiles are simultaneously performed and the results are shown in fig 3. Electromagnetic physics (EM) is turned on in TGLF for these simulations. The simulation profiles obtained by treating fast ions to be 'thermal' (corresponds to the 'Thermal' case shown in fig. 3) show clear underestimation for $\rho$ $<0.4$ while profile match is qualitatively improved (the ITB feature can be nicely reproduced) with beam ions treated as 'fast' ions (corresponds to the 'Fast' case shown in fig. 3). 'Thermal' ions share the temperature with main ions while 'fast' ions have their own temperature which is calculated by NUBEAM and is typically far larger.

To better understand the differences between the simulated profiles of 'thermal' and 'fast' cases, the temperature gradients, $a / L_{T e}\left(=a \nabla T_{e} / T_{e}\right)$ and $a / L_{T i}\left(=a \nabla T_{i} / T_{i}\right)$, for both cases are plotted in fig 3(c) and 3(d), respectively across the plasma volume. It can be seen that the predicted $a / L_{T}$ for both cases are nearly the same for $\rho>0.4$. For $\rho<0.28$, the black and magenta lines are closely aligned, suggesting that 'fast' ions do not play a major role. As $\rho$ approaches $\sim 0.33$, the black and magenta lines diverge violently, with only the latter showing the strong ITB observed in experiment. Clearly 'fast' ions are important for the ITB, but are they a necessary and sufficient condition? To answer this question, it is necessary to divide the inner region into two parts, namely inner core region $(\rho<0.28)$ and mid-core region $(\rho \sim 0.33)$, for separate discussion. 

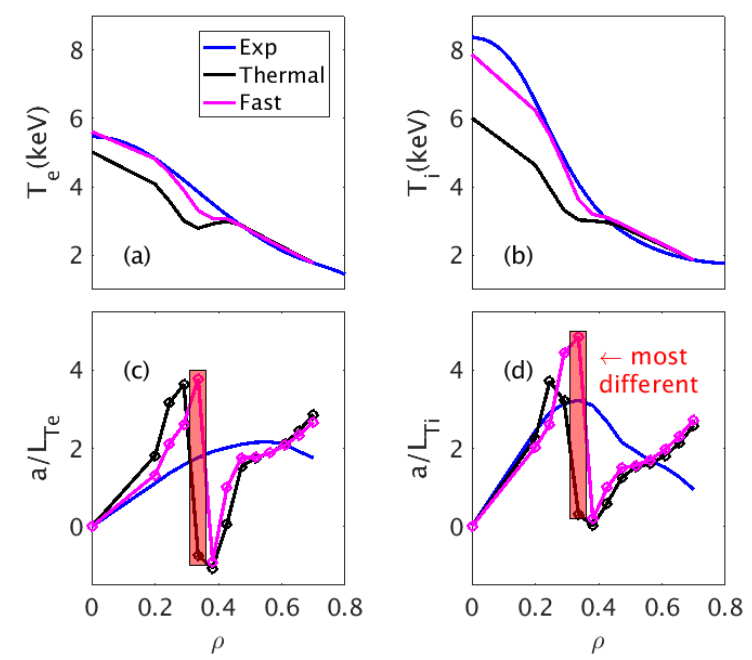

FIG 3. Profile comparison of (a) $T_{e}$, (b) $T_{i}$, (c) $a / L_{T e}$ and (d) $a / L_{T i}$, 'Exp' denotes the experimental profile. Simulation profiles with and without fast ions are denoted by 'Thermal' and 'Fast', respectively.

\subsection{EM and $\alpha$ effect}

Before moving on to detailed analysis, it is necessary to clarify what we mean by EM and $\alpha$ (stabilization) effects, which will be heavily used in this paper.

The EM effect is also called finite $\beta_{E}\left(=\frac{8 \pi n_{e} T_{e}}{B_{u n i t}^{2}}\right.$, where $B_{\text {unit }}$ is the effective field strength [28]) effect. It couples the ITG to KBM[29], which is a drift Alfvén mode, so that KBM can siphon some of the free energy from ITG and act to stabilize the ITG.

The $\alpha$ effect is essentially a geometric effect, it changes the particle magnetic drift velocity ( $v_{d}$, shown in Eq.(1)) to change the drift resonance and affect the mode stability[30].

$$
v_{d}=\frac{v_{\|}^{2}+\mu B}{\Omega_{c a} B} \vec{b} \times \nabla B+\frac{4 \pi v_{\|}^{2}}{\Omega_{c a} B^{2}} \vec{b} \times \nabla p=v_{d, \nabla B}+v_{d, \nabla p}
$$

The magnetic drift velocity $v_{d}$ in the gyrokinetic equation is composed of two terms [31], namely the drift induced by the inhomogeneity of toroidal magnetic field 
$\left(v_{d, \nabla B}\right)$ and the pressure gradient $\left(v_{d, \nabla p}\right)$, with the later essentially coming from the equilibrium magnetic field which is adjusted to balance the pressure gradient. The $v_{d, \nabla p}$ is proportional to $\alpha$, which can be expressed as

$$
\alpha=-q^{2} R \frac{8 \pi}{B_{\text {unit }}^{2}} \frac{d p}{d r}
$$

in a circular plasma, where $q, R$ and $p$ are the safety factor, major radius and pressure, respectively. Therefore, $\alpha$ can affect the mode stability via $v_{d, \nabla p}$ by changing the drift direction, thus detuning the drift resonance and reduces the turbulent growth rate. In addition to ITG, $\alpha$ effect can also stabilize the TEM and ETG as well as KBM. Please refer to the work of Beer et al[30] for more details on the physics mechanism of the $\alpha$ stabilization.

The term $\alpha$ is linearly proportional to $\beta_{E}$, which can be explicitly derived from Eq.(2),

$$
\alpha=-q^{2} \beta_{E} \frac{R}{a}\left[k_{i}\left(a / L_{T i}+a / L_{n i}\right)+\left(a / L_{T e}+a / L_{n e}\right)+k_{f}\left(a / L_{T f}+a / L_{n f}\right)\right],
$$

where $k_{i}=\frac{p_{i}}{p_{e}}, k_{f}=\frac{p_{f}}{p_{e}}$ and $p_{i}, p_{e}$ and $p_{f}$ are the pressure of thermal ions, electrons and fast ions, respectively. Therefore, increase of $\beta_{E}$ is favorable for both EM and $\alpha$ stabilization but their impacts are not the same. We note here that GYRO allows the evaluation of the EM and $\alpha$ effects separately by independently scaling the $\beta_{E}$ in the Ampere equation and the pressure-gradient drift velocity $v_{d, \nabla p}$ in the gyrokinetic equation, respectively in simulation, though $\beta_{E}$ and $\alpha$ are related by Eq.(3) in principle. It is noted that when we talk about the scaling of $\alpha$, essentially only $v_{d, \nabla p}$ is scaled and no attempt is made to change $\beta_{E}$ via Eq.(3), i.e. $\alpha$ stabilization can occur in the electrostatic (ES) limit. 


\subsection{Inner core region $(\rho<0.28)$}

For $\rho<0.28$, the predicted $a / L_{T}$ is quite high for both the 'thermal' and 'fast' case. Neoclassical transport dominates inside $\rho=0.28$ as shown in fig 4(a) since turbulence is heavily suppressed by the combination of rotation shear and electromagnetic (EM) stabilization [29]. The growth rate of ITG is significantly reduced by EM stabilization and is comparable to the $E_{r}$ shearing rate as shown in figure 4(b). The 'fast' case has higher $T_{e}$ which results in lower gyroBohm normalized target flux and therefore the predicted $a / L_{T}$ of thermal species for the 'fast' case is slightly lower, as shown in fig.3(c) and (d).

EM stabilization is important in turbulence suppression, as evidenced from comparing the growth rates at $\rho \sim 0.28$ [fig 4(b)]. To explore the conditions under which EM has the strongest effect, we take the ratio of the maximum growth rates of electrostatic and electromagnetic runs as an indicator of EM stabilization strength. The ratio increase with decrease of magnetic shear (figure 4(c)), indicating that lower magnetic shear is more favorable for EM stabilization.

$\beta_{E}$ acts to sophon the free energy from ITG to $\mathrm{KBM}$, therefore increase of $\beta_{E}$ is favorable for ITG suppression. However, on the other hand, KBM may thus be destabilized. This tendency can be clearly seen especially when the $\alpha$ effect is turned off (which means $v_{d, \nabla p}=0$ ), the KBM is predicted to be strongly unstable under experimental $\beta_{E}(\sim 0.011)$ (Fig 5(a)). In order to verify both modes, the eigenfunction of parallel magnetic potential $\left(\mathrm{A}_{\|}\right)$of both ITG and KBM are shown in fig 5(b), KBM clearly have $A_{\|}$of ballooning parity, which is not observed for ITG. However, KBM can be strongly suppressed by $\alpha\left(v_{d, \nabla p}\right)$, which is intrinsically accompanied with the $\beta_{E}$ as shown in Eq. (1). Fortunately, the KBM is fully stabilized for experimental $\alpha\left(\alpha_{\text {scale }}=1\right.$, fig 5(b)). From the ballooning mode theory, 
the KBM is likely entering the second stability region in the presence of high $\alpha$ and low magnetic shear in the experiment $[32,33]$ as shown in fig $5(\mathrm{c})$. A positive feedback loop emerges for the formation and sustainment of high performance plasma in the deep core. High $\beta_{E}$ can suppress the ITG by coupling to the KBM, leading to high $a / L_{T}$. The consequently large $\alpha$ (induced by high $\beta_{E}$ and $a / L_{T}$ ) in concert with low magnetic shear stabilizes the KBM by pushing it into the second-stable region to avoid the confinement degradation induced by KBM. Low magnetic shear is favorable for both the EM stabilization of ITG and $\alpha$ stabilization of KBM, and crucially important for the sustainment of such a loop. We note that the analysis above is based on a local gyrokinetic ballooning formulation. The conclusion that the KBM enters into the $2^{\text {nd }}$ stability region is, therefore, in the local sense. On the other hand, the low magnetic shear in the deep core region can make the mode radially extended (like to $\sim 10 \%$ of plasma radius for $k_{y} \rho_{s}=0.2$ at $\rho=0.33$ ), which may indicate that global effect needs to be considered. To accurately quantify the effect, one may need to run the global version of GYRO, which is extremely computationally intensive and will be left for a future study.
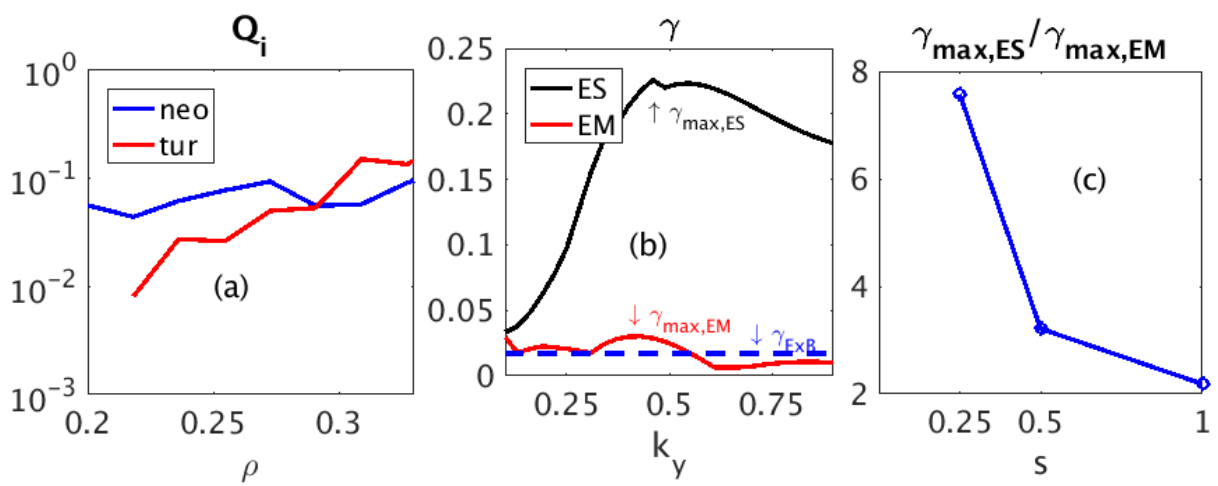

FIG 4 (a) Comparison of transport flux between neoclassical (blue) and turbulent (red) contribution for the 'thermal' case; (b) The linear growth rate at $\rho=0.28$ for electrostatic (ES, black line) and electromagnetic (EM, red line) TGLF run. The blue dashed line is the $E_{r}$ shearing rate; (c) the ratio of maximum growth rate of ES and 

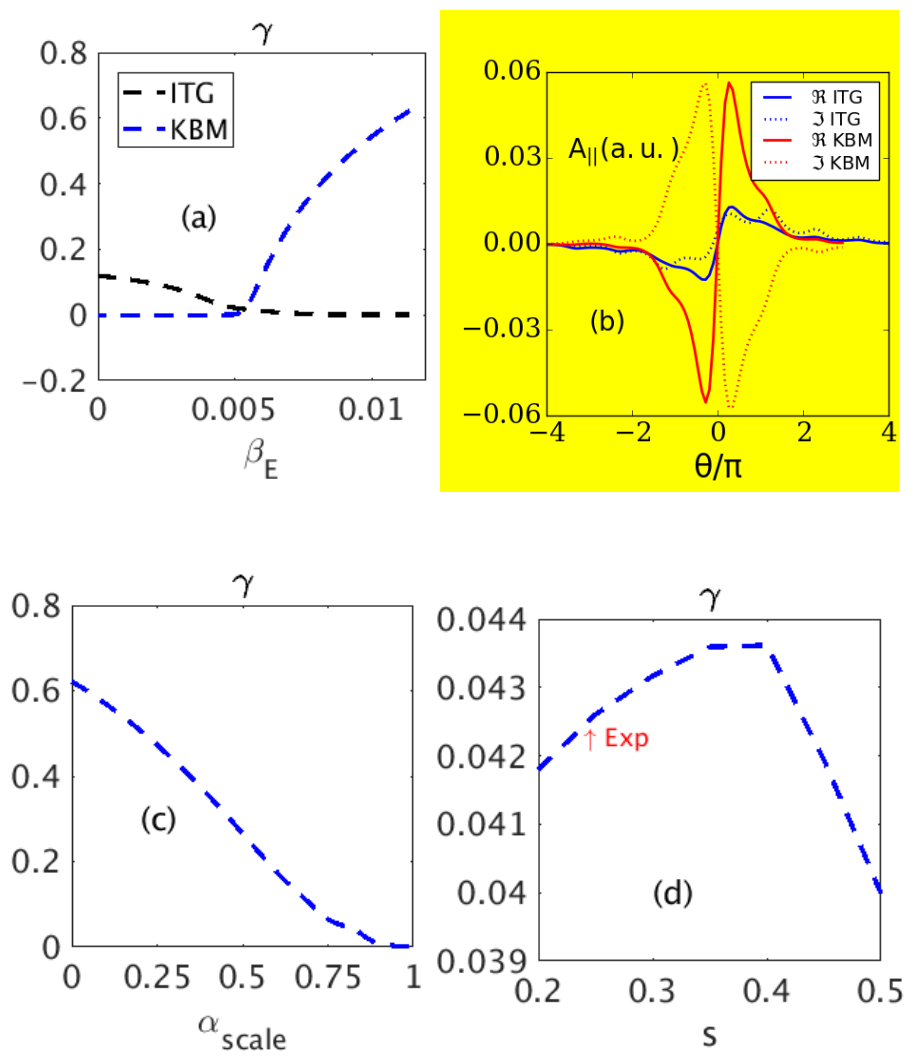

Fig 5 (a) The growth rate $\left(k_{y}=0.2\right)$ of ITG and KBM versus $\beta_{E}$ in the absence of $\alpha$ effect; (b) The eigenfunction of $A_{\|}$of both ITG and KBM shown in (a), real and imaginary part are shown in solid and dashed line, respectively. (c)The KBM growth rate versus scaling factor of experimental $\alpha$; (d) The KBM growth rate versus magnetic shear in the presence of $\alpha_{\text {scale }}=0.9 ;$ GYRO eigenvalue solver is used throughout this analysis

\section{4 mid-core region $(\rho \sim 0.33)$}

The largest difference between the 'fast' and 'thermal' case occurs near $\rho=0.33$, as covered by the red rectangular strip shown in fig 3 ; the 'fast' case has $\left(a / L_{T i}\right.$, $\left.a / L_{T e}\right)_{\mathrm{fast}}=(4.9,3.8)$ while 'thermal' case has $\left(a / L_{T i}, a / L_{T e}\right)_{\text {thermal }}=(0.3,-0.7)$. Since the simulated $T_{i}$ and $T_{e}$ profiles come from the integral of $a / L_{T i}$ and $a / L_{T e}$ over radius from the boundary towards the core, the higher $a / L_{T i}$ and $a / L_{T e}$ at $\rho=0.33$ for the 'fast' case allow the simulated profiles to match the experiment inside of $\rho=0.33$ and show the ITB feature, as opposed to the 'thermal' case .

To understand the huge discrepancies, the ion transport flux $Q_{i}$ versus $a / L_{T i}\left(a / L_{T e}\right.$ is fixed to be $\left.0.77 a / L_{T i}\right)$ at $\rho=0.33$ is shown in fig 6 with the other background parameters coming from experiment. The experimental flux is given by the blue 
dashed line, which intersects one point $\left(\mathrm{P}_{1}\right.$ in the figure) with the 'thermal' curve and three points (only the right one $\mathrm{P}_{2}$ is shown) with the 'fast' curve. $\mathrm{P}_{1}$ and $\mathrm{P}_{2}$ roughly correspond to the solution of 'thermal' and 'fast' case shown in fig 3, respectively. This explains the huge difference of the predicted $a / L_{T}$ between the 'fast' and 'thermal' cases. Another consequent two questions need to be understood for further interpretation, namely, (i) why the 'fast' case has lower flux so that it can intersect with the experimental flux at high $a / L_{T}$ ? This can be partly explained by the dilution effect induced by the fast ions, which has a fraction of $n_{f} / n_{e} \sim 14 \%$ which is significantly high; (ii) why the transport flux versus $a / L_{T i}$ shows a non-monotonic behavior?

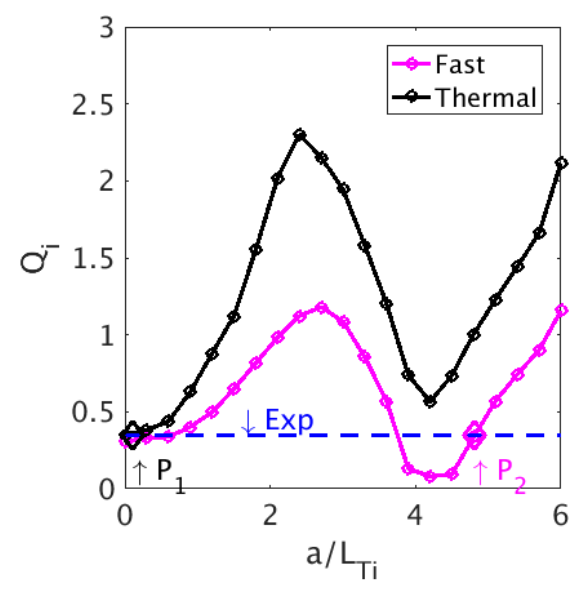

FIG 6. The predicted ion energy flux $Q_{i}$ versus $a / L_{T i}$ for the 'thermal' (black curve) and 'fast' (pink curve) case, $a / L_{T e}$ is set to be $0.77 a / L_{T i}$, calculated by TGLF-SAT0. Experimental flux is shown in blue dashed line

To answer question (ii), the $Q_{i}$ spectrum is plotted and shown to peak at $k_{y} \sim 0.1$ (fig 7(a)). Linear electrostatic analysis of the $k_{y}=0.1$ ITG mode using GYRO[24] with the $\alpha$ effect turned off and on (for the case 'on', $\alpha$ changes consistently with $a / L_{T i}$ ), is shown in fig 7(b). The growth rate with $\alpha$ 'on' shows a non-monotonic behavior with increasing $a / L_{T i}$, which is similar to the non-monotonic variation of the transport flux as depicted in fig 6. Such a characteristic does not exist with $\alpha$ 'off'. This strongly indicates that the non-monotonic behavior of the growth rate for the 'on' case, and consequently the flux, comes from the competing effects of the driving source $\left(a / L_{T i}\right.$ and $\left.a / L_{T e}\right)$ and $\alpha$ stabilization. Motivated by this, one would also expect 
that the higher $\alpha$ for the 'fast' case, which is $\sim 10 \%$ larger than the 'thermal' case, should also contribute to the lower flux for question (i).
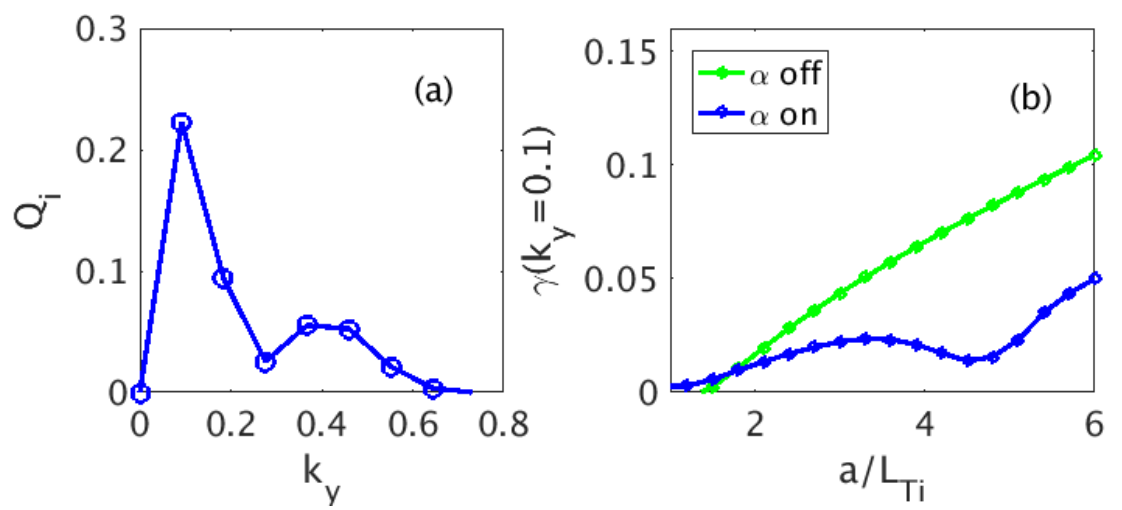

FIG 7. (a) $\mathrm{Q}_{\mathrm{i}}$ spectrum (TGLF-SAT0 result) for $a / L_{T i} \sim 2$ in Fig 6 . (b) The linear growth rate versus $a / L_{T i}$ with $\alpha$ effect on (blue) and off (green), $a / L_{T e}$ is fixed to be $0.77 a / L_{T i}$ (GYRO results).

The effectiveness of $\alpha$ stabilization can also be influenced by magnetic shear. Linear growth rates of ITG versus $a / L_{T i}$ for different magnetic shears $s$ are shown in fig 8 . We define the point of $a / L_{T i}$, beyond which $\alpha$ stabilization starts to 'win' over the driving term $a / L_{T i}$ and the growth rate starts to decrease, as the turning point. Both the growth rate and turning point decrease with $s$. In combination, they help to reduce the $a / L_{T i}$ threshold for $\alpha$ stabilization to affect confinement enhancement in low $\mathrm{s}$ condition. However, to quantitatively reproduce the experimental ITB, fast ion contribution has to be added as a third crucial element as highlighted in fig 6 .

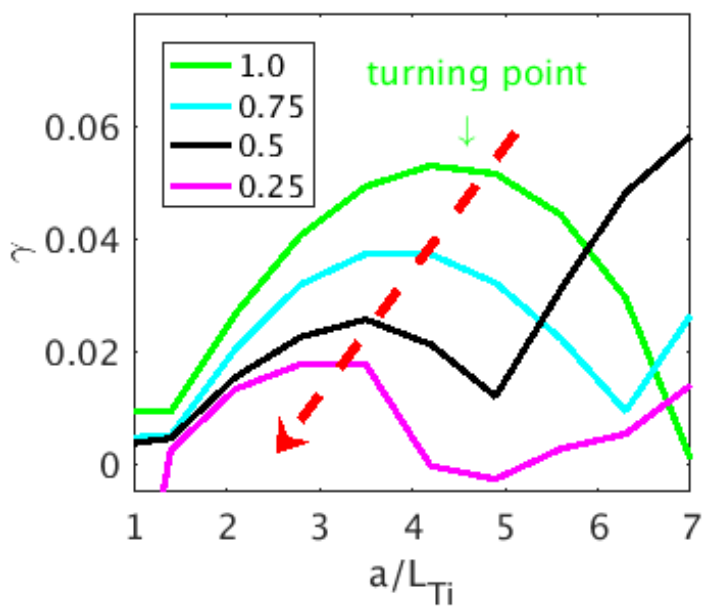

FIG 8. Growth rate $\gamma$ versus $a / L_{T i}$ with $\alpha$ effect on for different magnetic shears $s$, legend is $s . a / L_{T e}$ is 
fixed to be $0.77 a / L_{T i}$ (GYRO results).

\section{Summary and Conclusion}

To summarize, we have identified several dominant factors that work synergistically to produce confinement enhancement in the core region of hybrid mode experiments.

1. For $\rho<\sim 0.28$, the combination of EM stabilization and rotation shear can heavily suppress turbulent transport. Lower magnetic shear in this region is favorable for EM stabilization of ITG and pushes the KBM to the second stability region. Both aspects contribute to the formation and sustainment of peak temperature profile.

2. For $\rho \sim 0.33$, without fast ions, we cannot reproduce the ITB observed in experiment. The dilution effect induced by fast ions and the enhancement of $\alpha$ stabilization, which is more efficient with the low magnetic shear condition in the hybrid scenario, are crucially important for explaining the formation of ITB.

In conclusion, we demonstrate that a quasi-linear transport model is capable of quantitatively capturing the rich physics responsible for improved confinement in the core region of a hybrid discharge, identifiable by a broad flat $q$ profile region in the center. Low magnetic shear is favorable for $\alpha$ stabilization of both ITG and KBM as well as EM stabilization of ITG. Fast ions, which effectively suppress the turbulence by dilution effect and enhancement of $\alpha$ stabilization when $a / L_{T i}$ is high, provide the final element in ITB formation and the excellent profile match.

We note that previous simulations ${ }^{8}$ of this discharge using ASTRA-TGLF did not reach the large central ion temperatures. In these simulations, both the equilibrium and the $T_{i}$ profile were allowed to evolve, while the $T_{e}$ profile was fixed, unlike in the study presented here, where both temperature profiles are allowed to evolve, but the equilibrium is fixed. Applying the same boundary conditions to ASTRA-TGLF will 
reproduce the results shown here. Finally, we note that the rotation profile used in Ref [8] was also lower due to a misinterpretation of experimental data, but this alone cannot explain the difference.

Acknowledgement- Discussions with C. Holland, J. Candy, G. Staebler, J. Citrin and M. Romanelli are greatly appreciated. This work is supported by US DOE Office of Fusion Energy Science under Contract Number DE-SC0017992, China National Fusion Project for ITER under No.2017YFE0300500 and National Key R\&D Program of China Nos.

2017YFE0300500, 2017YFE0300501. Disclaimer: This report was prepared as an account of work sponsored by an agency of the United States Government. Neither the United States Government nor any agency thereof, nor any of their employees, makes any warranty, express or implied, or assumes any legal liability or responsibility for the accuracy, completeness, or usefulness of any information, apparatus, product, or process disclosed, or represents that its use would not infringe privately owned rights. Reference herein to any specific commercial product, process, or service by trade name, trademark, manufacturer, or otherwise does not necessarily constitute or imply its endorsement, recommendation, or favoring by the United States Government or any agency thereof. The views and opinions of authors expressed herein do not necessarily state or reflect those of the United States Government or any agency thereof.

1. Sips AC. Advanced scenarios for ITER operation. Plasma Physics and Controlled Fusion. 2005;47(5A):A19.

2. Garofalo AM, Gong X, Grierson BA, Ren Q, Solomon WM, Strait EJ, et al. Compatibility of internal transport barrier with steady-state operation in the high bootstrap fraction regime on DIII-D. Nuclear Fusion. 2015;55(12):123025.

3. Gormezano C, Sips A, Luce T, Ide S, Becoulet A, Litaudon X, et al. Steady state operation. Nuclear Fusion. 2007;47(6):S285.

4. Petty C, Kinsey J, Holcomb C, DeBoo J, Doyle E, Ferron J, et al. High-beta, steady-state hybrid scenario on DIII-D. Nuclear Fusion. 2015;56(1):016016.

5. Bock A, Fable E, Fischer R, Reich M, Rittich D, Stober J, et al. Non-inductive improved H-mode operation at ASDEX Upgrade. Nuclear Fusion.

2017;57(12):126041.

6. Petty CC, Nazikian R, Park JM, Turco F, Chen X, Cui L, et al. Advances in the steady-state hybrid regime in DIII-D — a fully non-inductive, ELM-suppressed 
scenario for ITER. Nuclear Fusion. 2017;57(11):116057.

7. Jardin S, Ferraro N, Krebs I. Self-organized stationary states of tokamaks. Physical review letters. 2015;115(21):215001.

8. Petty C, Austin M, Holcomb C, Jayakumar R, La Haye R, Luce T, et al.

Magnetic-flux pumping in high-performance, stationary plasmas with tearing modes. Physical review letters. 2009;102(4):045005.

9. Mantica P, Strintzi D, Tala T, Giroud C, Johnson T, Leggate H, et al.

Experimental study of the ion critical-gradient length and stiffness level and the impact of rotation in the JET tokamak. Physical review letters. 2009;102(17):175002. 10. Mantica P, Angioni C, Challis C, Colyer G, Frassinetti L, Hawkes N, et al. A key to improved ion core confinement in the JET tokamak: ion stiffness mitigation due to combined plasma rotation and low magnetic shear. Physical review letters.

2011;107(13):135004.

11. Citrin J, Jenko F, Mantica P, Told D, Bourdelle C, Garcia J, et al. Nonlinear stabilization of tokamak microturbulence by fast ions. Physical review letters. 2013;111(15):155001.

12. Doerk H, Bock A, Di Siena A, Fable E, Görler T, Jenko F, et al. Turbulence in high-beta ASDEX upgrade advanced scenarios. Nuclear Fusion. 2017;58(1):016044. 13. Sips A, Arslanbekov R, Atanasiu C, Becker W, Becker G, Behler K, et al. Steady state advanced scenarios at ASDEX Upgrade. Plasma Physics and Controlled Fusion. 2002;44(12B):B69.

14. Sips A, Tardini G, Forest C, Gruber O, Mc Carthy P, Gude A, et al. The performance of improved H-modes at ASDEX Upgrade and projection to ITER. Nuclear Fusion. 2007;47(11):1485.

15. Lao L, John HS, Stambaugh R, Kellman A, Pfeiffer W. Reconstruction of current profile parameters and plasma shapes in tokamaks. Nuclear fusion. 1985;25(11):1611. 16. Lao L, Ferron J, Groebner R, Howl W, John HS, Strait E, et al. Equilibrium analysis of current profiles in tokamaks. Nuclear Fusion. 1990;30(6):1035.

17. Fischer R, Bock A, Dunne M, Fuchs J, Giannone L, Lackner K, et al. Coupling of the flux diffusion equation with the equilibrium reconstruction at ASDEX Upgrade. Fusion Science and Technology. 2016;69(2):526-36.

18. Candy J, Holland C, Waltz R, Fahey MR, Belli E. Tokamak profile prediction using direct gyrokinetic and neoclassical simulation. Physics of Plasmas. 2009;16(6):060704.

19. Kinsey J, Staebler G, Waltz R. The first transport code simulations using the trapped gyro-Landau-fluid model. Physics of Plasmas. 2008;15(5):055908.

20. Belli E, Candy J. Kinetic calculation of neoclassical transport including self-consistent electron and impurity dynamics. Plasma Physics and Controlled Fusion. 2008;50(9):095010.

21. Houlberg W, Shaing K, Hirshman S, Zarnstorff M. Bootstrap current and neoclassical transport in tokamaks of arbitrary collisionality and aspect ratio. Physics of Plasmas. 1997;4(9):3230-42.

22. Waltz R, Staebler G, Dorland W, Hammett G, Kotschenreuther M, Konings J. A gyro-Landau-fluid transport model. Physics of Plasmas. 1997;4(7):2482-96. 
23. Kotschenreuther M, Rewoldt G, Tang W. Comparison of initial value and eigenvalue codes for kinetic toroidal plasma instabilities. Computer Physics Communications. 1995;88(2-3):128-40.

24. Candy J, Waltz R. An eulerian gyrokinetic-maxwell solver. Journal of Computational Physics. 2003;186(2):545-81.

25. Candy J, Waltz R. Anomalous transport scaling in the DIII-D tokamak matched by supercomputer simulation. Physical review letters. 2003;91(4):045001.

26. Miller R, Chu M, Greene J, Lin-Liu Y, Waltz R. Noncircular, finite aspect ratio, local equilibrium model. Physics of Plasmas. 1998;5(4):973-8.

27. Post DE, Jensen R, Tarter C, Grasberger W, Lokke W. Steady-state radiative cooling rates for low-density, high-temperature plasmas. Atomic data and nuclear data tables. 1977;20(5):397-439.

28. Candy J. A unified method for operator evaluation in local Grad-Shafranov plasma equilibria. Plasma Phys Control Fusion. 2009;51(10):105009.

29. Kim J, Horton W, Dong J. Electromagnetic effect on the toroidal ion temperature gradient mode. Physics of Fluids B: Plasma Physics. 1993;5(11):4030-9.

30. Beer MA, Hammett G, Rewoldt G, Synakowski E, Zarnstorff M, Dorland W.

Gyrofluid simulations of turbulence suppression in reversed-shear experiments on the Tokamak Fusion Test Reactor. Physics of Plasmas. 1997;4(5):1792-9.

31. Candy J, Belli E, Bravenec R. A high-accuracy Eulerian gyrokinetic solver for collisional plasmas. Journal of Computational Physics. 2016;324:73-93.

32. Staebler G, Garofalo A, Pan C, McClenaghan J, Van Zeeland M, Lao L. Transport barriers in bootstrap-driven tokamaks. Physics of Plasmas. 2018;25(5):056113.

33. Staebler GM. Theory of transport in high bootstrap fraction H-modes with internal transport barriers. Nuclear Fusion. 2018. 